\title{
Coilgun Acceleration Model Containing Multiple Interacting Coils
}

\author{
Kurt A. Polzin, Amanda B. Cipriano, and Adam K. Martin ${ }^{\ddagger}$ \\ NASA-George C. Marshall Space Flight Center, Huntsville, AL 35812 \\ Connie Y. Liu ${ }^{\S}$ \\ Georgia Institute of Technology, Atlanta, GA 30332
}

\begin{abstract}
A coilgun operates by pulsing current through an axially-arranged series of independently-controlled coils inductively interacting with a small, electrically-conductive, azimuthally-symmetric projectile to accelerate it to high velocities. The electrical circuits are programmed to pulse current through the coils in such a way so as to impart further electromagnetic acceleration in each stage. A method is developed to calculate the mutual inductance between the coils and between each coil and the projectile. These terms are used to write a system of first-order ordinary differential equations governing the projectile velocity and the current flow in each coil. While the inclusion of the electromagnetic interactions between coils significantly complicates the equation set as more coil sets are included in the problem, casting the problem symbolically in mass matrix form permits solution using standard numerical Runge-Kutta techniques. Comparing a projectile with a single-turn to that comprised of nine-turns, the inductance of the former is much smaller, but this leads to a greater induced projectile current. The lower inductance and greater current appear to offset each other with little difference in the acceleration profile for the two cases. For the limited cases studied, coils with a discharge half-cycle equal to the time for a projectile to transit from one coil to the next yield increased efficiency.
\end{abstract}

\section{Introduction}

$\mathrm{P}$ ULSED electromagnetic accelerators and launchers have been investigated because of their ability to propel payloads to high velocities, with significant effort having been expended studying a capacitor-driven concept known as a coilgun accelerator. ${ }^{1-4}$ In a coilgun (see schematic, Fig. 1), energy in a series of capacitors is discharged in sequence through inductive coils, producing a time-varying magnetic field according to Faraday's law that induces opposing currents in a conductive projectile and interacts with those induced currents to electromagnetically accelerate the projectile via the Lorentz force. The coilgun differs from the well-known railgun concept in that the acceleration in the former is accomplished entirely through electromagnetic field interactions between two (or more) separate current loops, while the accelerating projectile, or armature, in a railgun is required to complete the single high-current electrical discharge path.

In recent years, the coilgun has been investigated as a means of in-space electric propulsion. Systems of this type are of interest because, unlike other electric propulsion systems that must generate plasma, these inductive accelerators employ small cylindrical aluminum projectiles called macrons. The use of an already-conducting body alleviates the energy requirement to heat and ionize the propellant in plasma accelerators. In proof-of-concept testing, macrons massing $2 \mathrm{~g}$ have been accelerated in a multistage coilgun to the neighborhood of $400 \mathrm{~m} / \mathrm{s}$, and it is estimated that these systems could produce a range of specific impulses $\left(I_{\mathrm{sp}}\right)$ from $600-1000 \mathrm{~s} .{ }^{5,6}$ While electrothermal thrusters can operate in this regime, the frozen-flow losses associated with a thermal acceleration process can lead to lower efficiency relative to electromagnetic acceleration in a coilgun.

A coilgun-based propulsion system possesses the traditional advantages of using a pulsed system, such as the ability to operate using variable power levels and throttle the average thrust through adjustment of the pulsing rate. ${ }^{5}$

\footnotetext{
* Propulsion Research Scientist; Associate Fellow AIAA.

$\dagger$ NASA Pathways Intern

¥Physicist

$\S$ Graduate Research Assistant
} 


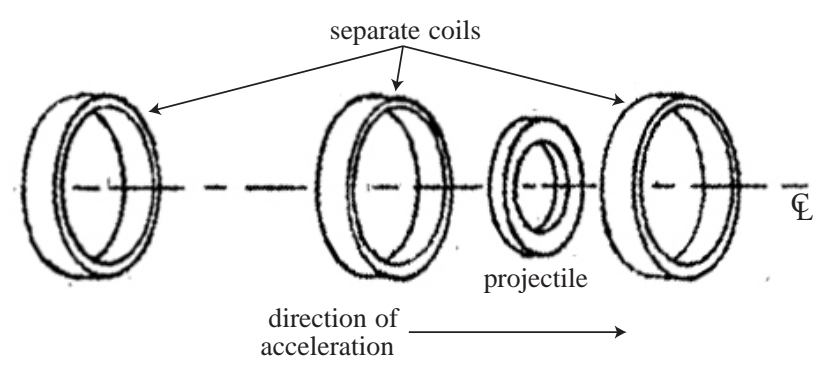

Figure 1. Schematic image of a multi-stage coilgun (after Ref. [1]).

The solid macrons also store as a solid as opposed to a high-pressure gaseous or liquid propellant. There are no physical or electrical connections to the projectile resulting in minimal barrel wear and degradation, with the reduction in frictional heating potentially permitting operation at higher repetition rates relative to railguns. ${ }^{5,7}$ The coils in a coilgun and/or the projectile can be comprised of multiple turns of conductor, yielding a greater mutual inductance between the two conducting loops and permitting greater transfer of magnetic field energy into acceleration of the projectile. In the instance of multiple, independently actuated acceleration coils, projectiles can be accelerated using a prearranged sequence of timed pulses to achieve greater velocities than what can be realized using only a single coil. The timing of this pulsing sequence can be better controlled using solid-state switching components, potentially increasing the power processing and energy recovery efficiencies. ${ }^{6}$

There has been significant experimental and modeling research on optimizing coilgun designs, ${ }^{7-11}$ but analysis and optimization of the coilgun geometry has proven to be a relatively complex undertaking. This is due to both the highly coupled nature of the equations governing the acceleration process and the electrodynamics of the system, and the time-varying nature of the mutual inductance between the coils and the projectile. Recent work has resulted in the identification of nondimensional scaling parameters, permitting a more systematic exploration of the parameter space of possible operating conditions. ${ }^{7}$

Owing to analytical difficulties, the modeling efforts to-date have been focused primarily on systems consisting of multiple, independent, non-interacting coils operating upon a projectile. However, the coils in a coilgun are typically close enough that if they are operating simultaneously, they should interact not only with the projectile but also with each other through the magnetic field. This situation of coils interacting with each other through a mutual inductance between each other makes the modeling significantly more complicated. The writing of a coupled set of circuit equations and an equation of motion for projectile acceleration in a multiple-stage coilgun is an involved but still analytically tractable problem. A difficulty arises in quantifying the mutual inductance between pairs of conductive coils and between the coils and the projectile as a function of projectile position. In this paper, we describe a method by which these terms can be determined for a system consisting of any number of coils and then as an illustration, present solutions to the model for a system consisting of five coils simultaneously interacting with one projectile. We solve the governing equations for varying accelerator stage capacitance and energy to demonstrate the effects varying these parameters have on acceleration and efficiency. This is, to our knowledge, the first work where the fully-coupled system of circuit equations was solved in the modeling of a coilgun.

In section II we present a finite element magnetic field modeling method for the multiple-stage coilgun configuration. We use this method to calculate the self-inductance and pairwise mutual inductance for each conducting body in the three-stage coilgun configuration. The acceleration model for a projectile interacting with an arbitrary number of independently-operating but electromagnetically-coupled coils is given in section III. Calculated results obtained by using the acceleration model are presented in section IV for two different projectile types, while further discussion of the work and potential future research is given in section V.

\section{Inductance Modeling}

The self-inductance of each coil and the mutual inductance between the coils and between each coil and the projectile as a function of projectile position were calculated through finite element magnetic field modeling with a 2-D axisymmetric magnetostatic solver (QuickField, Tera Analysis, Los Angeles, CA, USA). The program formulates the problem as Poisson's equation for a vector magnetic potential $\mathbf{A}$, where the magnetic field $\mathbf{B}=\nabla \times \mathbf{A}$, with either Dirichlet (used in this work) or Neumann boundary conditions. Poisson's equation is solved over an unstructured mesh through a successive over-relaxation method. The solution converges when the change in field energy between 

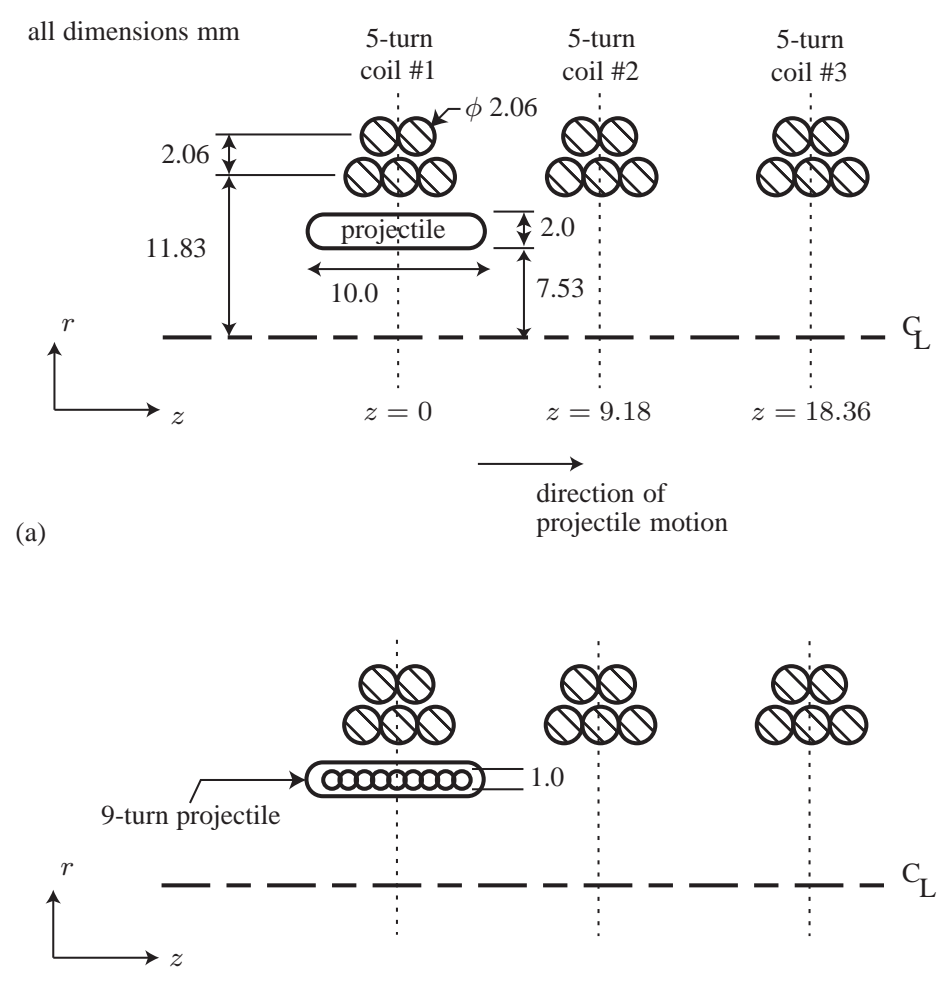

(b)

Figure 2. Two-dimensional axisymmetric schematic (not drawn to scale) of the finite element model used to simulate the magnetic field and compute the inductance profile for a system of three coils and one projectile (dimensions modeled from the experiment in Ref. [6]) where the projectile is modeled as (a) a single turn cylinder and (b) a 9-turn solenoid. (The projectile is at $z=0$ when it is symmetric about the the dashed line bisecting coil \#1.)

iterations at every point in the domain is below a user-defined threshold percentage. If the convergence threshold cannot be met during an iteration, the grid mesh is automatically refined.

A portion of the coil-projectile system employed in this study is schematically shown in Fig. 2 and is based on the five-turn coil geometry in Ref. [6]. In the present work, only 3 coils need to be magnetostatically modeled since, for the setup and dimensions of the problem, the mutual inductance and subsequent electromagnetic interaction between coils is negligible for separations greater than the distance between coils \#1 and \#3. The axisymmetric windings of each coil and of the projectile are modeled as conductors in series. DC current is permitted to flow in either one or two of the conductors at any time. As illustrated in the following discussion, this permits the deconvolution of the self-inductance and mutual inductance values between the various conductive coils.

We experimented with magnetic field modeling using either the magnetostatic or the AC magnetics solver within the finite element program. The AC magnetics solver yielded a more realistic solution of the current distribution within the conducting bodies as it captures nonuniformities owing to $1 / r$ effects, edge effects, and the frequencydependent interactions of currents flowing in the various conductors. Unfortunately, the current distribution in the AC magnetics case varies significantly between the modeling of a single conductor and that of multiple conductors interacting through the magnetic field because the coupled conductors mutually affect each other. While this is more physically realistic, the variation in the current density distributions for single coil versus multiple coupled coils alters substantially the the calculated self-inductance and mutual inductance calculated for the system This makes it impossible to independently model each conductor to calculate the self-inductance and then to find the mutual inductance terms through the linear superposition method employed in this paper. Using the magnetostatics method sacrifices the ability to capture $\mathrm{AC}$ magnetics effects, but it makes the determination of the inductance values for the system a more analytically-tractable problem. 


\section{A. Self-Inductance}

The magnetic field energy for current flowing in any isolated coil or in the projectile is obtained by first solving a finite element model for the resulting magnetic field produced by an arbitrary current flowing through the body. The magnetic field energy values of the solution for each cell within the domain are summed and the self-inductance of the isolated conductor $j$ is calculated as

$$
L_{j}=\frac{2 E_{\mathrm{M}}}{I_{j}{ }^{2}}
$$

where $I_{j}$ is the current in the $j$-th isolated conductor and $E_{\mathrm{M}}$ is the total energy stored in the magnetic field.

\section{B. Mutual Inductance}

The law of linear superposition applies to magnetic fields, so regardless of the number of conductors in a specific coilgun configuration, the magnetic fields of the system produced by currents in each coil will add linearly. This is mathematically written as

$$
\mathbf{B}(r, z)=\sum_{j=l}^{m} \mathbf{B}_{j}(r, z)+\mathbf{B}_{p}(r, z)
$$

where the summation is taken over the magnetic field contributions from coils $l$ through $m$, and the contribution to the magnetic field owing to the induced current in the projectile is given with a subscript $\mathrm{p}$. As an illustrative example, for a three coil system $(l=1, m=3$ ), the magnitude of the magnetic field at a given point can be written as (position coordinates omitted to simplify the notation)

$$
\begin{aligned}
|\mathbf{B}|^{2} & =\left|\mathbf{B}_{1}\right|^{2}+\left|\mathbf{B}_{2}\right|^{2}+\left|\mathbf{B}_{3}\right|^{2}+\left|\mathbf{B}_{\mathrm{p}}\right|^{2} \\
& +2 \mathbf{B}_{1} \cdot \mathbf{B}_{2}+2 \mathbf{B}_{1} \cdot \mathbf{B}_{3}+2 \mathbf{B}_{2} \cdot \mathbf{B}_{3} \\
& +2 \mathbf{B}_{1} \cdot \mathbf{B}_{\mathrm{p}}+2 \mathbf{B}_{2} \cdot \mathbf{B}_{\mathrm{p}}+2 \mathbf{B}_{3} \cdot \mathbf{B}_{\mathrm{p}}
\end{aligned}
$$

The stored inductive energy for such a setup can be written as

$$
\begin{aligned}
E_{\mathrm{M}} & =\frac{L_{1} I_{1}^{2}}{2}+\frac{L_{2} I_{2}^{2}}{2}+\frac{L_{3} I_{3}^{2}}{2}+\frac{L_{p} I_{\mathrm{p}}{ }^{2}}{2} \\
& +M_{12} I_{1} I_{2}+M_{13} I_{1} I_{3}+M_{23} I_{2} I_{3} \\
& +M_{1 \mathrm{p}} I_{1} I_{\mathrm{p}}+M_{2 \mathrm{p}} I_{2} I_{\mathrm{p}}+M_{3 \mathrm{p}} I_{3} I_{\mathrm{p}}
\end{aligned}
$$

where the mutual inductance $M_{x y}=M_{y x}$.

The self-inductance values of the three coils and the projectile were independently calculated in the previous section using Eq. (1). The pairwise mutual inductance terms can be obtained by modeling currents flowing in only two of the conductors at a time, running the simulation to obtain a magnetic field solution, and then summing the magnetic field energy over the model domain. This method is repeated until solutions for all the various pairwise conductor combinations have been generated, allowing for the calculation of all mutual inductance terms in Eq. (4). The situation is actually slightly easier in our case where the coils are all the same, and symmetry is useful in limiting the number of solutions that must be generated to fully quantify the mutual inductance terms. While the solutions were generated for the three-coil system of Fig. 2, we can expand upon these results to a more general $n$-coil system by neglecting the mutual inductance values for coil pairs separated by more than 2 coils (e.g., $M_{14} \approx 0$ ). For the five coil system modeled in this paper, the calculated self and mutual inductance values are given in Table 1.

The mutual inductance for the coil-projectile interaction is slightly more complicated because this value changes as the projectile moves axially. Consequently, the magnetic field model was solved many times for different projectile positions, producing the mutual inductance profile $M_{1 \mathrm{p}}(z)$ given in Fig. 3. Since the coils are all identical and separated by a distance $\Delta z=9.18 \mathrm{~mm}$, the mutual inductance profile for the coil-projectile is given by $M_{2 \mathrm{p}}(z)=M_{1 \mathrm{p}}(z-\Delta z)$, $M_{3 \mathrm{p}}(z)=M_{1 \mathrm{p}}(z-2 \Delta z)$, and so on. 
Table 1. Self and mutual inductance values for the five coil system modeled in this paper using the the geometry of Fig. 2. All other coil-to-coil mutual inductance values not given in this table are assumed equal to 0 .

\begin{tabular}{l|c}
\hline term & value $(\mathrm{nH})$ \\
\hline$L_{\mathrm{C} 1}$ & 752 \\
$L_{\mathrm{C} 2}$ & 752 \\
$L_{\mathrm{C} 3}$ & 752 \\
$L_{\mathrm{C} 4}$ & 752 \\
$L_{\mathrm{C} 5}$ & 752 \\
$L_{\mathrm{p}}$ (single turn) & 14.3 \\
$L_{\mathrm{p}}$ (nine turns) & 1299 \\
$M_{12}$ & 245 \\
$M_{23}$ & 245 \\
$M_{34}$ & 245 \\
$M_{45}$ & 245 \\
$M_{13}$ & 86 \\
$M_{24}$ & 86 \\
$M_{35}$ & 86 \\
\hline
\end{tabular}

\section{Acceleration Model}

A circuit-based model has already been developed to model projectile acceleration in coilguns and the acceleration process in pulsed inductive plasma thrusters. ${ }^{1,7,8,12-14}$ The 1-coil, 1-projectile model is modified in this section to account for additional coils and the electromagnetic interactions between coils.

\section{A. Circuit Equations}

For a general set of coils, numbered 1 to $N$, we assume that sequential coils $l$ through $m$ are operating at a given time. The coils outside of this range are assumed to be open circuits with infinite impedance that, consequently, do not affect the coils in which current is flowing. Shown in Fig. $4 \mathrm{a}$ is a lumped-element circuit representation of the $i$-th external coil circuit (for $l \leq i \leq m$ ). A corresponding circuit representation of the projectile and its interactions with the coils is given in Fig. 4b. The direction of the current arrows shows the direction of positive current flow.

The $i$-th external coil circuit possesses capacitance $C_{i}$, external inductance $L_{0 i}$, resistance $R_{\mathrm{e} i}$, and a coil self inductance $L_{\mathrm{C} i}$. A coil also couples to every other coil except itself through the mutual inductance terms $M_{i j}$. The projectile has a self inductance $L_{\mathrm{p}}$, a resistance $R_{\mathrm{p}}$, and it interacts with each coil individually through the positiondependent mutual inductance terms $M_{k \mathrm{p}}(z)$, for the set $\{k \mid l \leq k \leq m\}$.

Applying Kirchoff's law to the $i$-th current loop, we can obtain general equations for the system when sequential coils $l$ through $m$ have independent currents flowing through them. These equations can be written as:

$$
\begin{aligned}
\frac{d I_{i}}{d t} & =\left[V_{\mathrm{C}_{i}}-R_{\mathrm{e} i} I_{i}-\frac{d}{d t}\left(M_{i \mathrm{p}} I_{\mathrm{p}}\right)-\sum_{\substack{j=l \\
j \neq i}}^{m} M_{i j} \frac{d I_{j}}{d t}\right] /\left(L_{0 i}+L_{\mathrm{C} i}\right) \\
\frac{d V_{\mathrm{C} i}}{d t} & =-\frac{I_{i}}{C_{i}}
\end{aligned}
$$

where $V_{\mathrm{C} i}$ is the voltage on the capacitor in the $i$-th circuit. For all the coils that are not within the range from $l$ to $m$, the governing equations are given as

$$
\left.\begin{array}{l}
I_{n}, \frac{d I_{n}}{d t}=0 \\
\frac{d V_{\mathrm{C} n}}{d t}=0
\end{array}\right\} \text { for }\{n \mid n<l \vee n>m\}
$$



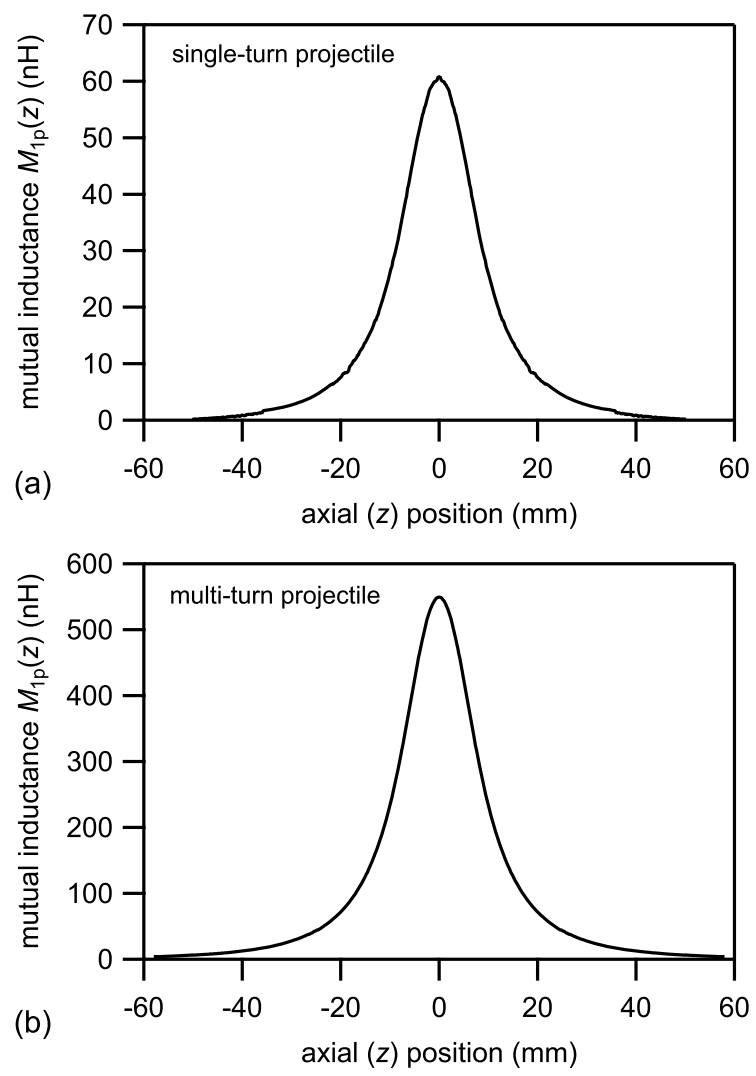

Figure 3. Coil-projectile mutual inductance profile $M_{1 \mathrm{p}}(z)$ for (a) the single-turn projectile and (b) the nine-turn projectile.

A single equation governing the projectile motion is written as

$$
\frac{d I_{\mathrm{p}}}{d t}=-\left[\sum_{k=l}^{m} \frac{d}{d t}\left(M_{k \mathrm{p}} I_{k}\right)+R_{\mathrm{p}} I_{\mathrm{p}}\right] / L_{\mathrm{p}} .
$$

The mutual inductance between each coil and the projectile is a function of the projectile's axial position $z$ as shown in Fig. 3. Within the system of equations the mutual inductance evolution between the $i$-th coil and the projectile is given as

$$
\frac{d M_{i \mathrm{p}}}{d t}=\frac{\partial M_{i \mathrm{p}}}{\partial z} \frac{d z}{d t}=\frac{\partial M_{i \mathrm{p}}}{\partial z} v_{\mathrm{z}}
$$

where $v_{\mathrm{z}}$ is the axial velocity of the projectile.

\section{B. Momentum Equation}

The momentum equation for this system is a statement of Lenz's law, summed over all the pairwise interactions between the active coils and the projectile. This can be compactly written as

$$
\begin{aligned}
\frac{d z}{d t} & =v_{\mathrm{z}} \\
\frac{d v_{\mathrm{z}}}{d t} & =\left(\sum_{k=l}^{m} I_{k} I_{\mathrm{p}} \frac{\partial M_{k \mathrm{p}}}{\partial z}\right) / m_{\mathrm{p}}
\end{aligned}
$$

where $m_{\mathrm{p}}$ is the mass of the projectile. 


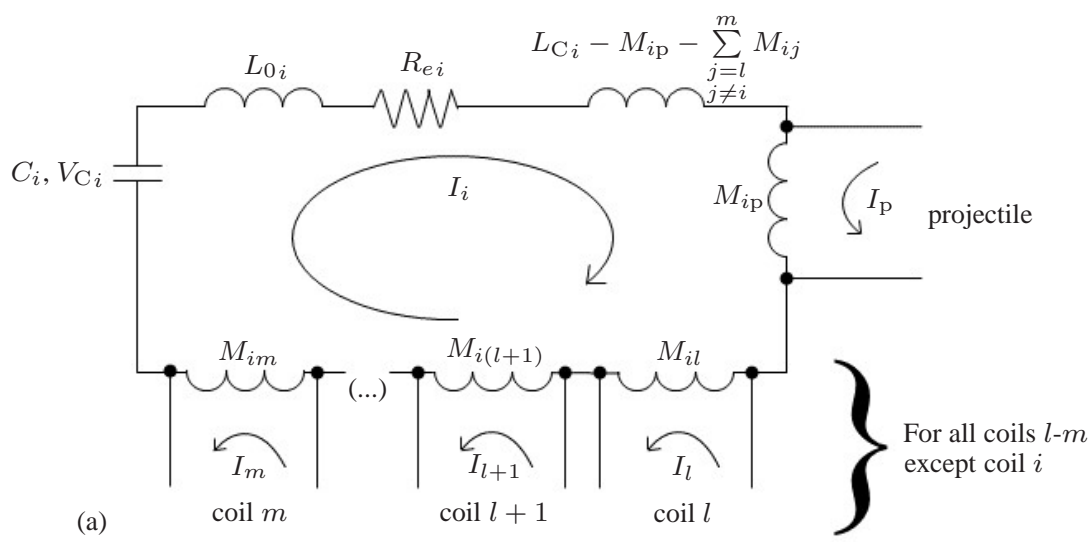

(a)

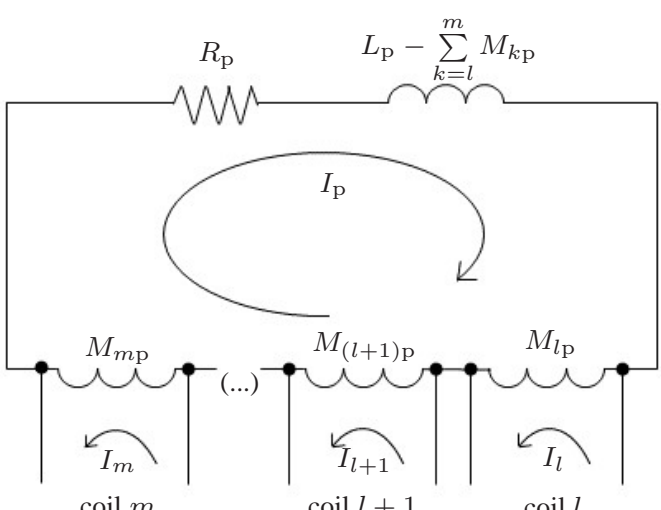

(b)

coil $m$

coil $l+1$

coil $l$

Figure 4. a) General lumped-element circuit schematic for interactions between the $i$-th current loop, the projectile, and all other active coils. b) Lumped-element circuit schematic showing the interactions between the projectile and all active, external coils.

\section{Calculated Results}

The model of the previous section can be used to calculate the acceleration of a projectile under various conditions and assumptions. The set consisting of Eqs. (5)-(7) for each coil and Eqs. (8)-(11) are integrated in time using a Runge-Kutta solver. The equation set is entered into Matlab in mass matrix form, which is generally written as

$$
\mathbf{M}(t, Y(t)) \cdot \mathbf{Y}^{\prime}(t)=\mathbf{F}(t, Y(t))
$$

where $\mathbf{Y}^{\prime}(t)$ is a general vector comprised of all the first order time derivatives in the set, $\mathbf{M}(t, Y(t))$ is the mass matrix of coefficients on the time derivatives, and the vector $\mathbf{F}(t, Y(t))$ consists of all the terms in the equation set not containing any time derivatives. The advantage in casting the problem in mass matrix form is that additional equations can be added later without the need to perform manual row-reduction to generate a system of first order ODEs where each equation contains only one time-derivative term, which is tedious even for the simple one coil-projectile problem. Instead, in the present work the row reductions are performed symbolically in Matlab.

The coil and projectile inductance properties were specified in Table 1 and Fig. 3. The assumed series resistance for each coil is $1 \mathrm{~m} \Omega$ while the assumed projectile resistance is $0.1 \mathrm{~m} \Omega$. A solution is generated by specifying the capacitance and initial charge voltage for each coil and the time current flow is initiated in each coil. For this paper, the projectile starting position is such that it is bisected by the $z=0$ line (see Fig. 2) and with an initial velocity $v_{z}(0)=20 \mathrm{~m} / \mathrm{s}$. For the given starting position, the initial velocity is needed because a perfectly centered projectile feels no accelerating force one way or the other since $\partial M_{1 \mathrm{p}} / \partial z(z=0)=0$.

During the course of the solution, the mutual inductance terms $\partial M_{i \mathrm{p}} / \partial z$ are numerically computed at each step using interpolation on the data set of Fig. 3. The interpolated mutual inductance values in the neighborhood of the projectile are used in a second-order-accurate finite difference routine to calculate the spatial derivative specific to the location of the projectile during an iteration. Subsequent coils are triggered as the projectile reaches them (i.e. as the projectile position aligns with the symmetry line for a coil set).

A summary of the test cases simulated and the conditions applied to the system is given in Table 2. The capacitance 
Table 2. Summary of stage voltages, capacitances, and energies for each simulated case.

\begin{tabular}{|l|c|c|c|c|c|c|}
\hline & \multicolumn{5}{|c|}{ single-turn projectile } & \multicolumn{3}{c|}{ nine-turn projectile } \\
& case 1 & case 2 & case 3 & case 4 & case 5 & case 6 \\
\hline & \multicolumn{5}{|c|}{ voltage (V) } \\
\hline stage 1 & 1700 & 1700 & 1700 & 1700 & 1700 & 1700 \\
stage 2 & -1700 & -1700 & -4543 & -1700 & -1700 & -5216 \\
stage 3 & -1700 & 1700 & 6853 & -1700 & 1700 & 7218 \\
stage 4 & -1700 & -1700 & -8341 & -1700 & -1700 & -8881 \\
stage 5 & -1700 & 1700 & 9943 & -1700 & 1700 & 10207 \\
\hline & \multicolumn{7}{|c|}{ capacitance $(\mu \mathrm{F})$} \\
\hline stage 1 & 660 & 650 & 650 & 660 & 685 & 685 \\
stage 2 & 660 & 126 & 91.0 & 660 & 104 & 72.8 \\
stage 3 & 660 & 90.0 & 40.0 & 660 & 84.0 & 38.0 \\
stage 4 & 660 & 69.8 & 27.0 & 660 & 69.0 & 25.1 \\
stage 5 & 660 & 69.0 & 19.0 & 660 & 73.0 & 19.0 \\
\hline & 950.7 & initial energy (J) \\
\hline stage 1 & 953.7 & 939.3 & 939.3 & 953.7 & 989.8 & 989.8 \\
stage 2 & 953.7 & 182.1 & 939.3 & 953.7 & 150.3 & 989.8 \\
stage 3 & 953.7 & 130.1 & 939.3 & 953.7 & 121.4 & 989.8 \\
stage 4 & 953.7 & 100.8 & 939.3 & 953.7 & 99.7 & 989.8 \\
stage 5 & 953.7 & 99.7 & 939.3 & 953.7 & 105.5 & 989.8 \\
\hline total & $\mathbf{4 7 6 9}$ & $\mathbf{1 4 5 2}$ & $\mathbf{4 6 9 6}$ & $\mathbf{4 7 6 9}$ & $\mathbf{1 4 6 7}$ & $\mathbf{4 9 4 9}$ \\
\hline
\end{tabular}

values and voltage magnitudes in cases 1 and 4 were held constant at values that approximately matched those used in the testing of Ref. [6] (note: in that work, only a single-turn projectile was used). Across all cases, the voltage on the first coil was kept constant. For cases 2 and 5, the voltage magnitude was kept constant for each stage and the capacitance was varied such that the projectile transited the distance between adjacent coils during one half-cycle of the coil current discharge. After transiting that distance, the next coil was triggered with a capacitance that resulted in a half-period matching the time for the projectile to transit to the next coil. This was repeated for all five coils. Because only the capacitance was varied, the energy per stage generally decreased from stage-to-stage. In cases 3 and 6 , the capacitance was varied in the manner of cases 2 and 5, such that the projectile would transit from one coil to the next during that coil's first half-cycle, but the discharge energy per coil stage was held constant throughout the problem. This resulted in much higher initial capacitor voltages for later stages.

Calculated results are presented in Fig. 5 for the single-turn projectile and in Fig. 6 for the nine-turn projectile. Initial stored energy, final projectile velocity and kinetic energy, and the efficiency converting stored energy to kinetic energy are summarized for each case in Table 3. The first thing we note is that, while the projectile inductance was significantly greater for the nine-turn projectile, other than restricting the value of the current in the projectile, the results are remarkably similar, especially with respect to the ultimate acceleration realized by the projectiles in comparable cases (1 and 4, 2 and 5, 3 and 6). The acceleration in Eq. (11) contains the projectile current and the

Table 3. Summary of energy transfer and efficiency for each simulated case.

\begin{tabular}{|l|c|c|c|c|c|c|}
\hline & \multicolumn{3}{|c|}{ single-turn projectile } & \multicolumn{3}{c|}{ nine-turn projectile } \\
& case 1 & case 2 & case 3 & case 4 & case 5 & case 6 \\
\hline initial energy (J) & 4769 & 1452 & 4696 & 4769 & 1467 & 4949 \\
final velocity (m/s) & 757 & 438 & 855 & 759 & 438 & 858 \\
final kinetic energy (J) & 572 & 192 & 731 & 576 & 192 & 736 \\
efficiency (\%) & 12.0 & 13.2 & 15.6 & 12.1 & 13.1 & 14.9 \\
\hline
\end{tabular}



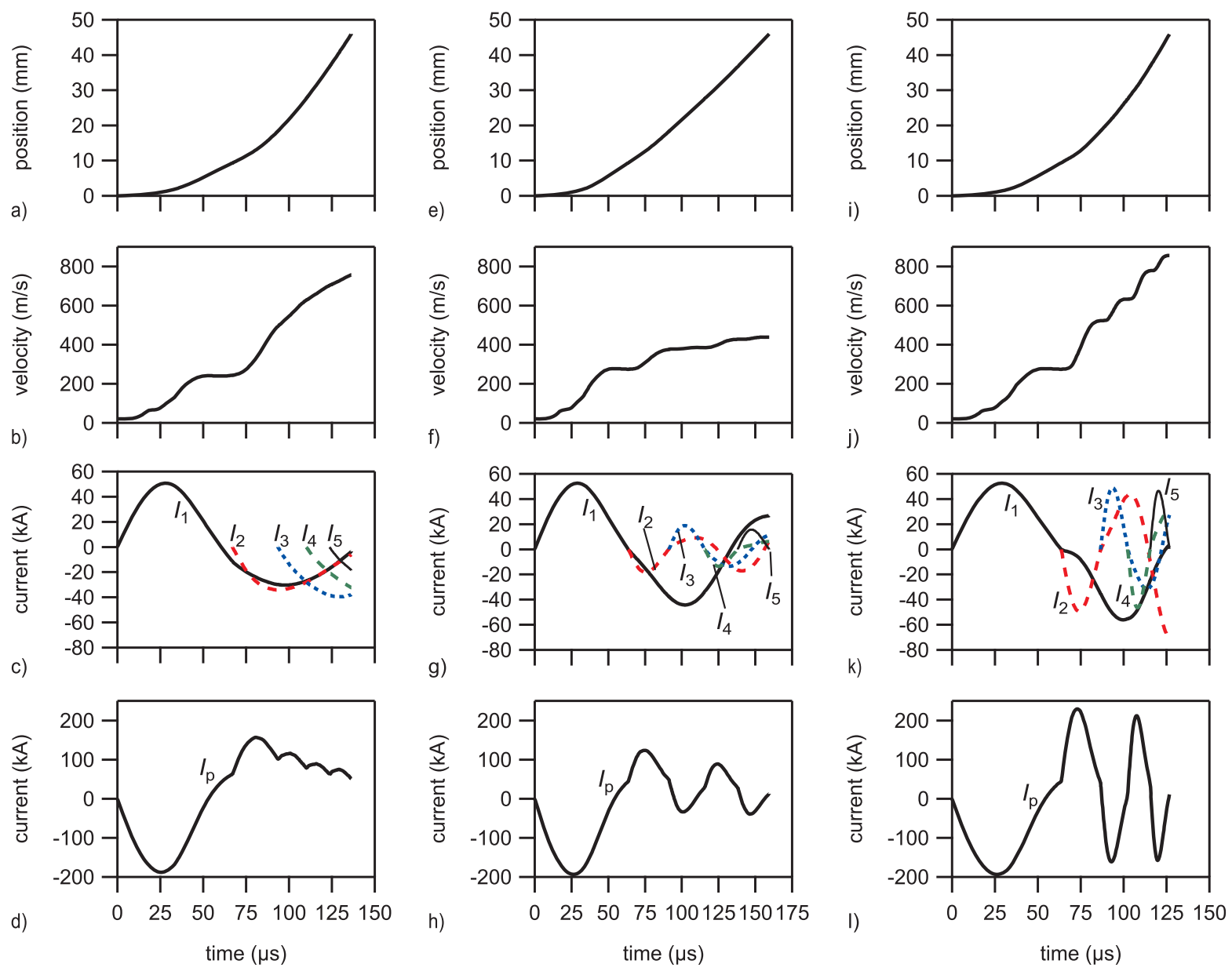

Figure 5. Calculated single-turn projectile position, projectile velocity, individual coil currents, and projectile current as a function of time for: (a)-(d) case 1, (e)-(h) case 2, (i)-(l) case 3.

spatial derivative of the mutual inductance with respect to time. For the single-turn projectile, the former is large while the latter is small, while for the nine-turn projectile the reverse is true. In terms of net acceleration, the changes in these two terms appear to largely offset each other.

For the baseline cases 1 and 4, the voltages on the later stage were all maintained as negative values. Examining the current waveforms we see that positive voltages for later stages (stage 3, for instance) would result in a significant period of time while the projectile was near to the third coil set where the currents $I_{3}$ and $I_{p}$ were in the same direction. Since the spatial derivative of the mutual inductance is negative, this would cause Eq. (11) to yield a negative acceleration during part of the discharge, significantly reducing the overall energy transferred. To obtain maximum acceleration in these cases, the voltages for stages 2-5 were all kept negative.

For cases $2,3,5$, and 6 , the voltage varied from positive to negative for each stage, since the projectile mostly oscillates in the same manner as the coil to which it is closest with the value of $I_{\mathrm{p}}$ nearly zero when the next coil is triggered. The efficiency of the energy transfer process is greater when the capacitor values are adjusted such that each coil discharges through one-half cycle during the projectile transit time from one coil to the next. In addition, we observe in Table 2 that the capacitance to match the circuit with the intra-coil transit time is affected by the voltage applied to the coil. This is due to additional acceleration realized in cases 3 and 6 owing to the higher voltages and commensurately higher current levels in coils $2-5$, reducing the transit time between coils. Finally, it can be noted that the highest efficiencies and exit velocities are realized for the cases where the first half-cycle of the circuit is matched with the transit time for the projectile and where the discharge energy per stage is held constant.

Much effort was expended to ensure that the coil-to-coil mutual inductance values and their effects on the coil currents were properly captured. This effect can, in particular, be observed in Figs. 5 or 6, plots (g) and (k). For the 

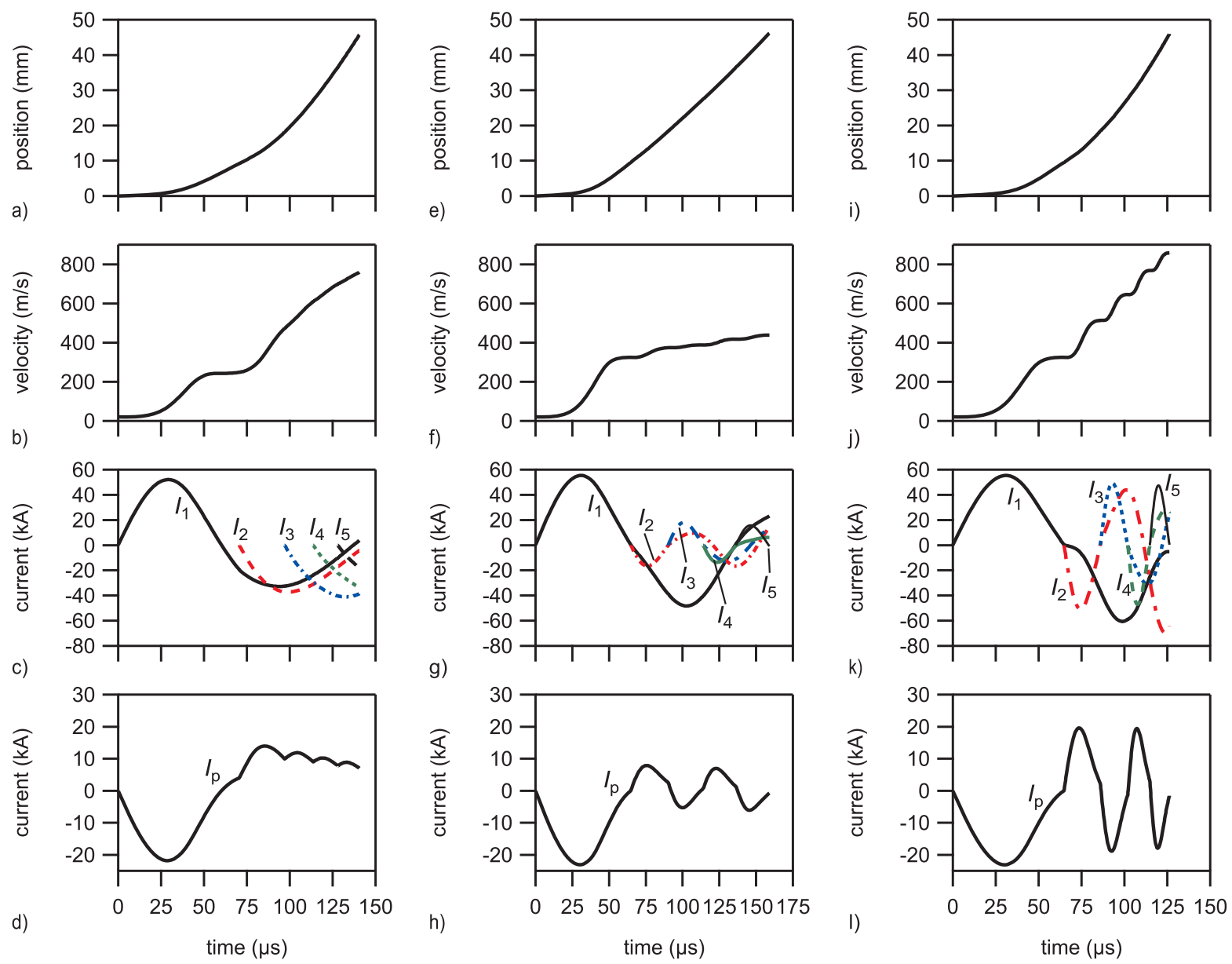

Figure 6. Calculated nine-turn projectile position, projectile velocity, individual coil currents, and projectile current as a function of time for: (a)-(d) case 1, (e)-(h) case 2, (i)-(l) case 3.

case in plot (k) where the initial energy for coil 2 is much greater, we observe a significant deviation of current $I_{1}$ when $I_{2}$ is initiated. Even in plot (g), it can be observed that the slope of $I_{1}$ changes somewhat when coil 2 is triggered.

\section{Future Work}

While we demonstrate in this work that the acceleration efficiency can be improved by adjusting the stage capacitance to alter the external circuit ringing frequency, the present results are insufficient to claim that this is a globally optimized solution. We also observed that maintaining the discharge energy yielded an improvement on efficiency and resulted in a much higher exhaust velocity. However, it should be noted that the baseline cases 1 and 4, which were not optimized at all, produced results (projectile velocities, accelerator efficiencies) that were comparable to those obtained in the match circuit cases.

The solutions generated in this paper for a small subset of the parameter space reveal the close-coupled nature of the problem. While we have examined some of the effects associated with varying stage capacitance and voltage, this work is by no means a complete study and significant work remains to fully quantify these effects. Furthermore, we recognize that there are several additional parameters that could be varied in the design and optimization of a coilgun. These include a more arbitrary variation of the pulse timing for each stage beyond simply activating a coil when the projectile is directly under it, changing of the projectile start position and initial velocity, and the potential use of switches that can 'turn off' (such as gated turn-off thyristors), which could interrupt the current in coils reducing the coupling in the problem and saving/recovering energy that could be used to accelerate ensuing projectiles. The stage and mutual inductance values can also be varied, although that is much harder to vary without significant additional 
magnetostatic modeling associated with every geometric configuration of interest.

\section{Conclusions}

We have presented solutions to an acceleration model for a coilgun that includes the mutual inductance coupling between individual coils of the gun. Magnetic field modeling of the system of coils and the projectile was used to calculate individual magnetic field contributions for each component, allowing for the determination of both the self and mutual inductance values for the setup. Calculated results generated for a single-turn and nine-turn projectile produced several insights and conclusions.

- A system of first-order ordinary differential equations governing the momentum of a projectile and the currents in each coil of a coilgun can be written for an arbitrary number of coils electromagnetically coupled to the projectile and to each other through their mutual inductance terms. The system can be put into a mass matrix form, symbolically manipulated, and solved numerically using a Runge-Kutta routine. The present work represents, to our knowledge, the first time such a system has been solved with the inclusion of coil-to-coil mutual inductance terms.

- The single-turn and nine-turn projectiles have significantly different inductance values that result in a large disparity in the induced projectile current levels, but the net acceleration for the two different projectiles is remarkably similar.

- For the cases simulated, efficiency increased as the capacitance was adjusted to match the half-period of each coil with the projectile transit time from one coil to the next.

- All other things remaining equal, increasing the discharge energy per stage generally resulted in correspondingly greater acceleration imparted to the projectile.

- While these results represent first of their kind simulations for a coupled-coil or self-coupled coilgun, the solutions were obtained over a very narrow sliver of the operational coilgun parameter space. Until further explored, the maximizations described in this paper should not be taken as an overall optimization of projectile acceleration or energy transfer efficiency.

\section{References}

${ }^{1}$ McKinney K and Mongeau P 1984 IEEE Trans. Magnetics 20239

${ }^{2}$ Kaye R J, Cnare E C, Cowan M, Duggin B W, Lipinski R J, Marder B M, Douglas G M, and Shimp K J 1993 IEEE Trans. Magnetics 29680

${ }^{3}$ Yanjie C, Wenbiao L, Ruifeng L, Yi Z, and Bengui Z 2009 IEEE Trans. Magnetics 45518

${ }^{4}$ Wenbiao L, Yanjie C, Zhang Y, Wang J, and Yang D 2011 IEEE Trans. Plasma Sci. 39100

${ }^{5}$ Kirtley D, Slough J, Schonig J, and Ketsdever A $20104^{\text {th }}$ Joint Army-Navy-NASA-Air Force (JANNAF) Spacecraft Propulsion Subcommittee Meeting (Colorado Springs, $\mathrm{CO}$ )

${ }^{6}$ Pancotti A, Kirtley D, and Slough J $20115^{\text {th }}$ Joint Army-Navy-NASA-Air Force (JANNAF) Spacecraft Propulsion Subcommittee Meeting (Huntsville, $A L$ )

${ }^{7}$ Polzin K A, Adwar J, and Hallock A K 2013 IEEE Trans. Magnetics 491453

${ }^{8}$ Kim S-W, Jung H-K, and Hahn S-Y 1996 IEEE Trans. Magnetics 32505

${ }^{9}$ Marder B 1993 IEEE Trans. Magnetics 29701

${ }^{10}$ Guo L, Guo N, Wang S, Qiu J, Zhu J G, Guo Y, and Wang Y 2009 IEEE Energy Conversion Congress and Exposition (San Jose, CA)

${ }^{11}$ Zabar Z, Naot Y, Birenbaum L, Levi E, and Joshi P N 1989 IEEE Trans. Magnetics 25627

${ }^{12}$ Kaye R J 2005 IEEE Trans. Magnetics 41194

${ }^{13}$ Polzin K A and Choueiri E Y 2006 IEEE Trans. Plasma Sci. 34945

${ }^{14}$ Polzin K A, Sankaran K, Ritchie A G, and Reneau J P 2013 J. Phys. D: Appl. Phys. 46475201 